\title{
Contests with a Stochastic Number of Players
}

\author{
Wooyoung Lim and Alexander Matros* \\ Department of Economics \\ University of Pittsburgh
}

September 17, 2008

\begin{abstract}
We study Tullock's (1980) $n$-player contest when each player has an independent probability $0<p \leq 1$ of participating. A unique symmetric equilibrium is found for any $n$ and $p$ and its properties are analyzed. In particular, we show that for a fixed $n>2$ individual equilibrium spending as a function of $p$ is single-peaked and satisfies a single-crossing property for any two different numbers of potential players. However, total equilibrium spending is monotonically increasing in $p$ and $n$. We also demonstrate that ex-post overdissipation is a feature of the pure-strategy equilibrium in our model. It turns out that if the contest designer can strategically decide whether to reveal the actual number of participating players or not, then the actual number of participants is always revealed.
\end{abstract}

Keywords: Contests, Stochastic number of players, Over-dissipation.

JEL classification: C72, D72, D82.

${ }^{*}$ We thank the associate editor and three anonymous referees for helpful comments. We are grateful for useful discussions and suggestions from Michael R. Baye, Oliver Board, Tim Hister, Sergei Izmalkov, Kai Konrad, Daniel J. Kovenock, Jonathan Lafky, Ernest K. Lai, Michael Menietti, Roger Myerson, Johannes Münster, Jack Ochs, Shmuel Nitzan, Karl Wäneryd; seminar and workshop participants at University of Adelaide, University of Nevada, University of New South Wales, University of Pittsburgh, the New Economic School, the workshop "Contests and Tournaments" at the Social Science Research Center Berlin (WZB), the first international conference of "Tournaments, Contests and Relative Performance Evaluation," and the Third World Congress of the Game Theory Society. 


\section{Introduction}

Contest theory, which started from Tullock's famous paper, ${ }^{1}$ standardly assumes that the number of players is fixed. ${ }^{2}$ However, sometimes players do not know the actual number of players participating in the contest. For example, in many rent-seeking contests an individual lobbyist does not know how many other lobbyists are competing for the rent when she exerts her effort. ${ }^{3}$ In this paper, we consider Tullock's $n$-player contest where each player has an independent probability $0<p \leq 1$ of participation.

We show that such contests have a unique symmetric equilibrium which can be described in closed form. We analyze properties of this equilibrium and compare individual and total equilibrium spending in the standard Tullock model and in our case. Note that the standard Tullock model is a special case of our model where the participation probability is one (i.e. $p=1)$. Individual equilibrium spending, $X^{*}(r, V, n, p)$, depends on four parameters: the marginal return, the prize value, the number of potential players, and the probability of participation. It turns out that individual equilibrium spending is strictly increasing in the marginal return and the value of the prize for any fixed $n$ and $p$. Therefore, total equilibrium spending is also strictly increasing in the same parameters. These observations are, of course, consistent with the standard Tullock model where $p=1$.

We show that individual equilibrium spending as a function of the participation probability $p$ is single-peaked. Moreover, for any number of potential players $n$, the unique positive probability which maximizes individual equilibrium spending is always strictly less than one. Therefore, each active player spends more under some uncertainty about the number of players than under certainty. There are several reasons why it is important to know about individual equilibrium spending. Sometimes the contest outcome depends solely on the winner's individual spending. In many R\&D races, for instance, losing firms end up with no right to produce so their effort and investments are socially wasteful. In this case, total spending is not important but the individual spending made by the winning firm is. Our analysis suggests that it can be beneficial for society to have some degree of uncertainty in this case.

We also demonstrate that a single-crossing property ${ }^{4}$ holds for individual equilibrium

\footnotetext{
${ }^{1}$ Models similar to Tullock's (1980) have been studied in the literature on advertising and rivalry for market shares. Friedman's (1958) paper is probably one of the first in the literature.

${ }^{2}$ Surveys of the contest literature can be found in Nitzan (1994), Szymanski (2003), Congleton, et al. (2007), and Konrad (2007).

${ }^{3}$ The same situation often takes place in $R \& D$ races when firms do not know the actual number of $R \& D$ race competitors. Another example is a standard lottery where one player wins a unique main prize. Typically, when players buy lottery tickets they do not know the actual number of players.

${ }^{4}$ This property is different from the Spence's single crossing condition that has an important role in signaling, contract theory, and mechanism design.
} 
spending: for a fixed marginal return and a prize value, any two individual equilibrium spending curves (as functions of $p$ ) with different potential numbers of players cross only once. This property is surprising and important. First, the single-crossing property helps to identify the unique probability which maximizes individual equilibrium spending: We prove that two adjacent individual equilibrium spending curves cross at the peaked point of the curve with a higher number of potential players. Second, based on the single-crossing property, we show that the interval of participation probabilities is divided into two parts. If $p>0.9$, then the standard contest result that individual equilibrium spending is decreasing in the number of participating players holds. However, if $0<p<0.9$, then the above result does not hold.

It turns out that the comparative statics are much simpler for total equilibrium spending than individual equilibrium spending. Although individual equilibrium spending is strictly increasing in only two out of four parameters, total equilibrium spending is strictly increasing in all four parameters. In particular, unlike individual equilibrium spending, ex-ante total equilibrium spending (as a function of probability $p$ ) is maximized under certainty when $p=1$.

Our model provides another possible answer for a long-standing question about overdissipation in equilibrium. Since Krueger (1974), Posner (1975), and Tullock (1980), there have been many attempts including Corcoran (1984), Corcoran and Karels (1985), Higgins, et al.(1985), Michaels (1988), and Leininger and Yang (1994) to explain over-dissipation within a theoretical framework. Now it is a well-known result that ex-ante over-dissipation is not consistent with equilibrium behavior. ${ }^{5}$ Ex-ante over-dissipation never takes place in our model either. Since total equilibrium spending is monotonically increasing in $p$, the highest expected (ex-ante) total spending is achieved in Tullock's case $p=1$.

Hillman and Samet (1987) and Baye, et al. (1994, 1999) show that ex-post over-dissipation can take place as a particular realization of a mixed-strategy equilibrium. We demonstrate that ex-post over-dissipation is a natural feature of the pure-strategy equilibrium if the number of players is stochastic. The intuition for this observation is straightforward. If the participation probability is below one, $p<1$, the actual number of players in the contest can be different from the expected number of players. We demonstrate that if $n>3$ and the actual number of players is much higher than the expected number of players, then ex-post over-dissipation occurs. However, the expected number of players always coincides with the actual number of players if $p=1$ and, therefore, ex-post over-dissipation never takes place in that case.

Games with an uncertain or stochastic number of players are a natural extension of games

\footnotetext{
${ }^{5}$ Recently, Baharad and Nitzan (2008) have shown that over-dissipation is possible in equilibrium, when the contestants distort their winning probabilities.
} 
with a fixed number of players. In auction theory, McAfee and McMillan (1987) were the first to study models with a stochastic number of bidders. They show that standard auction theory is sensitive to the assumption that the number of players is common knowledge. Levin and Smith (1994) consider auctions with stochastic entrants resulting from endogenous entry. They extend the revenue-equivalence and ranking theorems and show that the seller and society can benefit from policies that reduce market thickness. Levin and Ozdenoren (2004) investigate bidders' and sellers' responses to ambiguity about the number of bidders in standard auctions with independent private valuations. They show that the general revenue equivalence result breaks down under ambiguity about the number of bidders. Myerson (1998) develops a mathematical framework to analyze games with population uncertainty and shows special properties of Poisson games. ${ }^{6}$

Myerson and Wärneryd (2006) and Münster (2006) are the first to consider contests with a stochastic number of players. Myerson and Wärneryd (2006) analyze a model with infinitely many potential players where the number of players is a random variable. They show that if it is known for certain that there will be at least one participant, then total equilibrium spending is strictly lower in a contest with population uncertainty than in a non-uncertain contest with the same expected number of players. In our model, the number of players is a random variable which follows the binomial distribution. Even though we do not require that there has to be at least one participant in the contest, we are also able to demonstrate the same result as in Myerson and Wärneryd (2006). Münster (2006) considers a model similar to ours. However, he focuses on the players' risk attitudes and shows that equilibrium rent seeking efforts are lower under risk aversion if and only if the expected fraction of active contestants is low.

Finally, we analyze a situation when the contest designer privately learns the actual number of players in the contest. It turns out that ex-ante the contest designer and the players are indifferent whether the information about the actual number of participants is revealed or not. Moreover, if the contest designer can strategically reveal his private information, the number of actual players is always revealed in equilibrium.

The rest of the paper is organized as follows. In the next section, we set up the model and describe the unique symmetric equilibrium. We focus on the properties of individual equilibrium spending in Section 3. Section 4 is devoted to the properties of total equilibrium spending. Information revelation about the actual number of players is analyzed in Section 5 . We conclude in Section 6. All proofs are in the Appendix.

\footnotetext{
${ }^{6}$ In a Poisson game, the number of players is a random variable which follows the Poisson distribution.
} 


\section{The Model}

Consider a contest with $n$ potential risk neutral players. We assume that each potential player participates (becomes active) in the contest with an independent probability $p \in(0,1]$. All active players compete for a single prize of value $V$. The timing of the game is as follows. First, nature chooses the active players. Then, without knowing the actual number of participants, each active player $i$ makes an expenditure, denoted by $X_{i} \geq 0$. The winner of the contest is determined by the following contest success function

$$
P_{i}\left(X_{i} ; \mathbf{M}\right)=\left\{\begin{array}{cc}
\frac{1}{|\mathbf{M}|}, & \text { if } \quad X_{j}=0 \text { for all } j \in \mathbf{M}, \\
\frac{X_{i}^{r}}{X_{i}^{r}+\sum_{j \in \mathbf{M}} X_{j}^{r}}, & \text { otherwise, }
\end{array}\right.
$$

where $r>0, \mathbf{M}$ is the set of active players in the contest, and $|\mathbf{M}|$ denotes the cardinality of M. If player $i$ participates in the contest, she maximizes the following objective function

$$
\max _{X_{i}} V \cdot\left(\sum_{\mathbf{M} \in \mathcal{P}^{\mathbf{N}_{i}}} p^{|\mathbf{M}|}(1-p)^{\left|\mathbf{N}_{i} \backslash \mathbf{M}\right|} P_{i}\left(X_{i} ; \mathbf{M}\right)\right)-X_{i},
$$

where $\mathbf{N}_{i}$ is the set of player $i$ 's possible opponents and $\mathcal{P}^{\mathbf{N}_{i}}$ is the set of all subsets of $\mathbf{N}_{i}$.

The first order condition for player $i$ is

$$
V \cdot\left(\sum_{\mathbf{M} \in \mathcal{P} \mathbf{N}_{i}} p^{|\mathbf{M}|}(1-p)^{\left|\mathbf{N}_{i} \backslash \mathbf{M}\right|} \frac{r X_{i}^{r-1} \sum_{j \in \mathbf{M}} X_{j}^{r}}{\left(X_{i}^{r}+\sum_{j \in \mathbf{M}} X_{j}^{r}\right)^{2}}\right)-1=0 .
$$

We focus on a symmetric equilibrium where $X_{1}=\ldots=X_{n}=X^{*}$. Then, the first order condition (3) becomes

$$
X^{*}=r V \cdot\left(\sum_{\mathbf{M} \in \mathcal{P}^{\mathbf{N}_{i}}} p^{|\mathbf{M}|}(1-p)^{\left|\mathbf{N}_{i} \backslash \mathbf{M}\right|} \frac{|\mathbf{M}|}{(|\mathbf{M}|+1)^{2}}\right) .
$$

Note that there are $C_{|\mathbf{M}|}^{n-1}$ different ways to make a set which has exactly $|\mathbf{M}|$ elements from the set $\mathbf{N}_{i}$. Using this fact, we can rewrite the individual spending (4) as follows

$$
X^{*}=r V \cdot\left(C_{n-1}^{n-1} p^{n-1} \frac{(n-1)}{n^{2}}+\ldots+C_{1}^{n-1} p^{1}(1-p)^{n-2} \frac{1}{2^{2}}\right)
$$

where $C_{j}^{n-1}=\frac{(n-1) !}{j !(n-j-1) !}$.

Now we can state the main result of this section.

Theorem 1. Suppose that $0<r \leq \frac{n+1}{n}$. Then, there exists a unique symmetric pure-strategy equilibrium where each player's expenditure is

$$
X^{*}(r, V, n, p)=r V \cdot\left(\sum_{i=1}^{n-1} C_{i}^{n-1} p^{i}(1-p)^{n-i-1} \frac{i}{(i+1)^{2}}\right) .
$$


It is easy to see now that the original Tullock model is a special case of our model where all players participate in the contest with probability 1 . Thus, we get the following corollary.

Corollary 1 (Tullock's individual spending). If $0<r \leq \frac{n+1}{n}$ and $p=1$, then

$$
X^{*}(r, V, n, 1)=\frac{(n-1)}{n^{2}} r V .
$$

\section{Individual Spending}

In this section we examine the impact of each parameter on individual equilibrium spending, $X^{*}(r, V, n, p)$, and show that $p$ and $n$ have non-monotonic effects on individual equilibrium spending.

First, we consider marginal return, $r$, and prize value, $V$. It is straightforward to see from (5) that individual equilibrium spending is strictly increasing in both $r$ and $V$. This means that Tullock's $(p=1)$ finding that individual equilibrium spending is a strictly increasing function of marginal return and prize value is robust to introducing a stochastic number of players.

Consider next the remaining parameters, the number of players, $n$, and the participation probability, $p$. Note that if there are only two potential players, then individual equilibrium spending is monotonic (strictly increasing) in the probability of participation, $p$, because

$$
X^{*}(r, V, 2, p)=\frac{r V}{4} p .
$$

However, our next example demonstrates that individual equilibrium spending is not monotonic in the probability of participation for $n \geq 3$.

Example 1. Suppose that $n=3$ and $r=V=1$. Then

$$
X^{*}(1,1,3,0.8)=0.222<X^{*}(1,1,3,0.9)=0.225>X^{*}(1,1,3,1)=0.222 .
$$

This example shows that individual equilibrium spending can be higher if there is some degree of uncertainty $(p<1)$ than under certainty $(p=1)$. The following theorem demonstrates that this observation is true in general: individual equilibrium spending is single-peaked in $p$.

Theorem 2 (Single-Peaked Property). For any $n \geq 3$, there exists a unique $p^{*}(n) \in(0,1)$ that maximizes individual equilibrium spending. Moreover, for given $r, V$, and $n, X^{*}(r, V, n, p)$ is single-peaked in $p$. 
In order to see the intuition behind this property, consider individual equilibrium spending, expression (5), which can be rewritten in the following way

$$
X^{*}(r, V, n, p)=\left(\sum_{i=1}^{n} q(i-1 ; p) x(r, V, i)\right),
$$

where $x(r, V, i)=\frac{i-1}{i^{2}} r V$ is individual equilibrium spending in the contest with $i$ actual players and $q(i ; p)=C_{i}^{n-1} p^{i}(1-p)^{n-i-1}$ is the probability that an active player faces exactly $i$ opponents in the contest (there are $i+1$ players in the contest). In other words, individual equilibrium spending, (8), is a weighted average of individual equilibrium spending in the case of $1,2,3, \ldots, n$ actual players, where the corresponding weight represents the binomial density assigned to the number of actual opponents of the active player. Note that if an active player faces zero opponents, then she is the only player in the contest and her equilibrium spending is zero (see the success function (1)). Moreover, it is a well-known result that individual equilibrium spending decreases (if $i \geq 2$ ) in the number of players in the contest without uncertainty. Therefore,

$$
x(r, V, 2)>x(r, V, 3)>\ldots>x(r, V, n-1)>x(r, V, n)>x(r, V, 1)=0 .
$$

Note that if $p$ is very small (very close to zero) in (8), then most of the weight is given to the first coefficient, $x(r, V, 1)=0$, so that individual equilibrium spending is also very small. Now if $p$ increases, most of the weight shifts from the first to the second coefficient $x(r, V, 2)$ and individual equilibrium spending increases. Finally, if $p$ increases more, most of the weight shifts from the second coefficient to coefficients three, four and so on. Since the second coefficient is the biggest among all the coefficients, individual equilibrium spending will decrease. ${ }^{7}$ Figure 1 illustrates the intuition for $n=4 .^{8}$

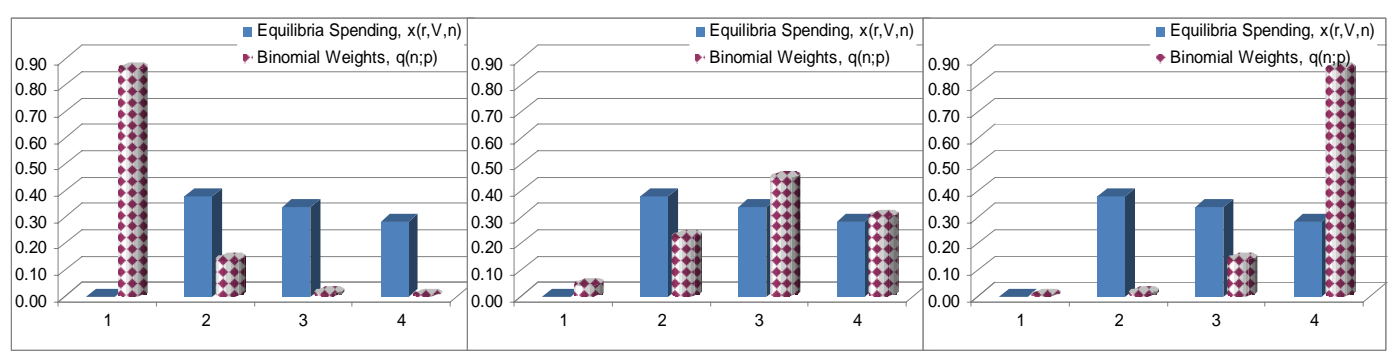
Figure 1: (a) small $p$
(b) $p=p^{*}$
(c) large $p$

\footnotetext{
${ }^{7}$ Note that the very last effect is absent if $n=2$. Hence, individual equilibrium spending is monotonically increasing in $p$ if $n=2$.

${ }^{8}$ Note that $r$ and $V$ are chosen such that $x(r, V, 1)+x(r, V, 2)+x(r, V, 3)+x(r, V, 4)=1$.
} 
The Single-Peak Property means that for any given $n$ there exists a unique participation probability, denoted by $p^{*}(n)$, that maximizes individual equilibrium spending. How will this probability change if $n$ increases? In order to investigate that, we need to understand the relationship between two different individual spending curves with different numbers of potential players. The following identity demonstrates the connection between two adjacent individual spending curves.

Theorem 3. Suppose that $n \geq 3$, then

$$
(n-1)\left(X^{*}(r, V, n, p)-X^{*}(r, V, n-1, p)\right) \equiv p \cdot \frac{\partial X^{*}(r, V, n, p)}{\partial p} .
$$

Since the right-hand side of (10) can be equal to zero only at $p^{*}(n)$ from the single-peak property, the individual equilibrium spending curves $X^{*}(r, V, n, p)$ and $X^{*}(r, V, n-1, p)$ can only intersect once if $p \in(0,1)$. Moreover, the intersection point must be the peak point of the curve $X^{*}(r, V, n, p)$. This gives the following corollary:

Corollary 2. For all $n \geq 2$, curve $X^{*}(r, V, n, p)$ intersects curve $X^{*}(r, V, n+1, p)$ at the peak point of $X^{*}(r, V, n+1, p)$.

The following theorem states even stronger results that $p^{*}(n)$ and $X^{*}\left(r, V, n, p^{*}(n)\right)$ decrease as $n$ increases. These results are derived directly from the single-peak property and identity (10).

Theorem 4. Suppose that $r$ and $V$ are given. Then, i) $p^{*}(n)$ strictly decreases as $n$ increases;

ii) $X^{*}\left(r, V, n, p^{*}(n)\right)$ strictly decreases as $n$ increases.

Let us examine how individual equilibrium spending changes if $n$ increases. In the original Tullock (1980) model, individual equilibrium spending is a strictly decreasing function of the number of players. However, the following example shows that this is not the case under uncertainty.

Example 2. Suppose that $p=0.8$ and $r=V=1$. Then

$$
X^{*}(1,1,2,0.8)=0.2<X^{*}(1,1,3,0.8)=0.2222>X^{*}(1,1,4,0.8)=0.2053 .^{9}
$$

When do we have a non-monotonic relationship between $n$ and individual equilibrium spending? Does the presence of some degree of participation uncertainty always guarantee this non-monotonicity? To answer these questions, we first show that any two individual equilibrium spending curves with different number of potential players cross only once on the interval $p \in(0,1]$.

\footnotetext{
${ }^{9}$ Münster (2006) also points this possibility out.
} 
Theorem 5 (Single-Crossing Property). For any $2 \leq m<n$, there exists a unique $p(m, n) \in$ $(0,1]$ such that

$$
X^{*}(r, V, m, p(m, n))=X^{*}(r, V, n, p(m, n)) .
$$

The intuition for the single-crossing property is as follows. Consider two different numbers of potential players $2 \leq m<n$. If $p$ is very small (very close to zero) in (8), most of the weight, $(1-p)^{m-1}$ and $(1-p)^{n-1}$, is given to the first coefficient, $x(r, V, 1)=0$, in the weighted average. Since $2 \leq m<n$, individual equilibrium spending is higher for $n$ potential players (it puts less weight on the first coefficient). If $p$ is very close to one, individual equilibrium spending in our model becomes very close to what it would be in Tullock's model and hence is higher for $m$ potential players. Since individual equilibrium spending is continuous in $p$, it has to be a "crossing" point for two individual equilibrium spending curves with different numbers of potential players. Theorem 5 shows that such a crossing point is unique.

Theorems 4 and 5 demonstrate that the individual equilibrium spending curves do not intersect to the right of $p^{*}(3)$. This implies that we observe the non-monotonic relationship between $n$ and individual equilibrium spending if and only if the participation probability is smaller than $p^{*}(3)=0.9$.

Corollary 3. Suppose that $r$ and $V$ are given. Then the individual equilibrium spending, $X^{*}(r, V, n, p)$, is monotonically decreasing in $n$ if and only if $0.9<p \leq 1$.

Corollary 3 describes the whole range of $p$ where Tullock's monotonicity result holds: more players lead to less individual spending. The intuition behind this result is as follows: Since there is monotonicity in the extreme case $p=1$ and individual equilibrium spending is continuous in the parameter $p$, monotonicity should hold in some neighborhood of the Tullock's model. Corollary 3 describes this neighborhood. Note that (from the single-crossing property) if $p$ is smaller than $p^{*}(3)=0.9$ (the crossing point of the individual spending curves with $n=2$ and $n=3$ ), individual spending with three potential players is higher than the individual spending with two potential players. This means that monotonicity no longer holds when $p<0.9$. All results of this section are summarized in Figure 3.

\section{Total Spending}

In this section, we examine the impact of each parameter on total equilibrium spending. Even though in the previous section we showed that individual equilibrium spending is not monotonic in $p$ and $n$, here we demonstrate that total equilibrium spending is indeed monotonic in all parameters. Next, we show that ex-post over-dissipation can occur. Finally, we discuss properties of total equilibrium spending if the expected number of players, $p n$, is fixed. 


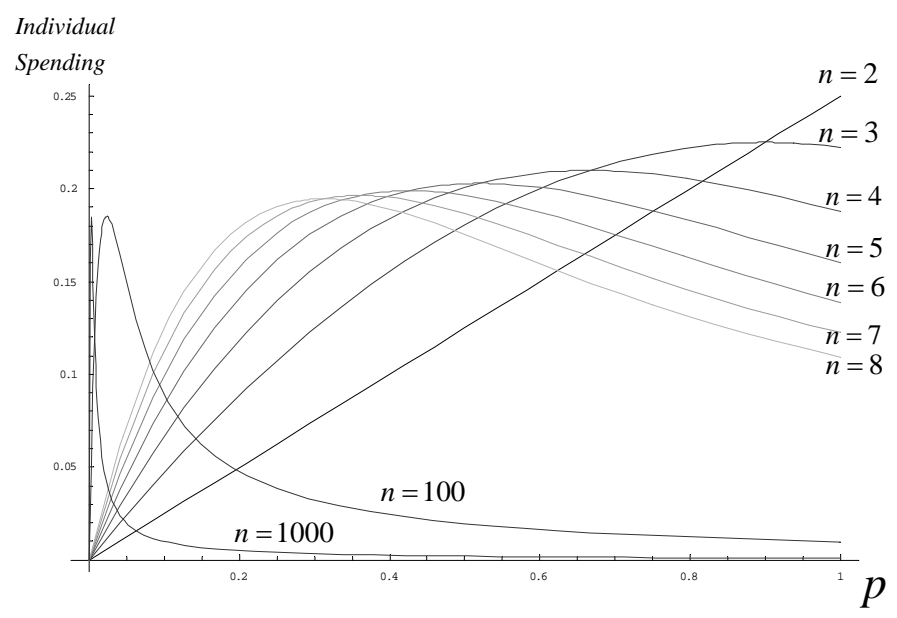

Figure 2: Equilibrium individual spending when $r V=1$.

\subsection{Properties}

Let $N^{*}$ and $T^{*}$ denote the ex-ante expected number of players and the ex-ante total equilibrium spending, respectively. Then

$$
N^{*}=C_{n}^{n} p^{n} n+C_{n-1}^{n} p^{n-1}(1-p)(n-1)+\ldots+C_{1}^{n} p(1-p)^{n-1} 1=n p .
$$

Note that the expected number of players is the same as an expectation of the random variable that follows a binomial distribution $B(n, p)$ where the probability of success is $p$ and the number of trials is $n$.

The expected total equilibrium spending is

$$
T^{*}(r, V, n, p)=n p \cdot X^{*}(r, V, n, p)=r V \cdot\left(\sum_{i=1}^{n} C_{i}^{n} p^{i}(1-p)^{n-i}\left(1-\frac{1}{i}\right)\right) .
$$

From equation (13), it is straightforward to see that the total spending increases as either $V$ or $r$ increases.

Consider now how a participation probability and the number of potential players affect total equilibrium spending. We will only discuss the effect of the participation probability here. Similar reasoning can be applied to the number of potential players. From Theorem 2, it is clear that for a small $p<p^{*}(n)$ the expected number of players and individual equilibrium spending will both increase if $p$ increases. Therefore, total equilibrium spending will increase too. However, if $p \geq p^{*}(n)$, then an increase of $p$ has two different effects on the expected total equilibrium spending. First, it always increases the expected number of players (see equation (12)). Second, it decreases individual equilibrium spending. Note that the second effect gets 


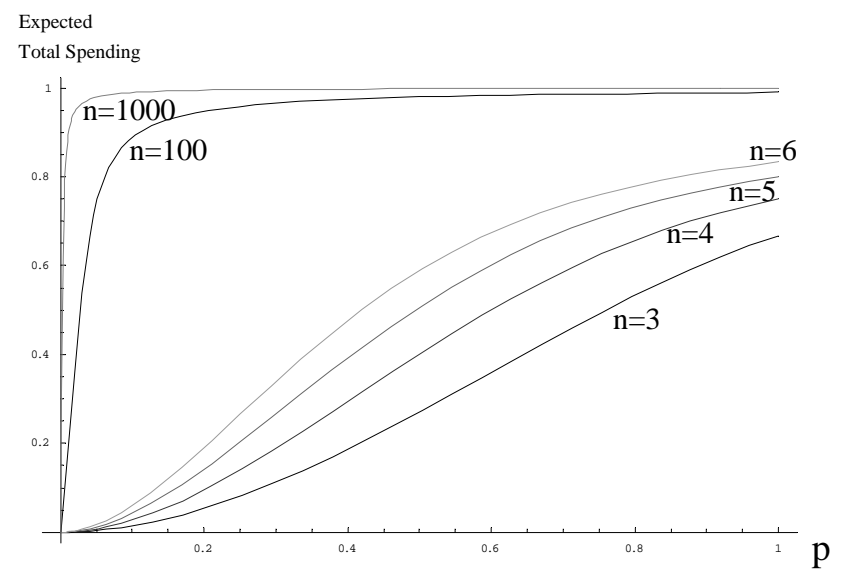

Figure 3: Expected total equilibrium spending. $r V=1$.

stronger as $p$ increases. In the extreme case when $p=1$ the second effect reaches its peak. However, as is well-known in the literature, even in this case the first effect is stronger. The following theorem states this formally.

Theorem 6. Suppose that $r$ and $V$ are given. Then,

i) for any $n \geq 2$, the expected total equilibrium spending increases as $p$ increases;

ii) for any $p \in(0,1]$, the expected total equilibrium spending increases as $n$ increases.

Figure 3 illustrates Theorem 6.

\subsection{Over-dissipation}

In this subsection, we show that if the actual number of players in the contest is "much higher" than the expected number of players, then ex-post over-dissipation can occur.

The possibility of ex-post over-dissipation is reminiscent of a similar observation in auction literature. For example, Levin and Smith (Proposition 3, 1994) study auctions with stochastic entrants and show that entry would be excessive from social and private points of view in the common-value auction.

Denote $R T^{*}(r, V, n, p ; k)$ as actual total spending, where $k$ is the actual number of players. Then in the symmetric equilibrium,

$$
R T^{*}(r, V, n, p ; k)=k \cdot X^{*}(r, V, n, p) .
$$

Ex-post over-dissipation takes place if and only if actual total spending exceeds the prize value, 
$V$. That is,

$$
R T^{*}(r, V, n, p ; k)=k \cdot X^{*}(r, V, n, p)>V .
$$

From (14) and (5), it follows that ex-post over-dissipation occurs if

$$
n \geq k>k^{*}:=\frac{1}{r \cdot\left(\sum_{i=1}^{n-1} C_{i}^{n-1} p^{i}(1-p)^{n-i-1} \frac{i}{(i+1)^{2}}\right)} .
$$

It is clear from (15) that ex-post over-dissipation never happens if the critical number $k^{*}$ is greater than the number of potential players $k^{*} \geq n$. This happens for small numbers of potential players, $n=2,3$. For example, if $n=3$, we get

$$
V>0.9 V=R T^{*}(4 / 3, V, 3,0.9 ; 3) \geq R T^{*}(r, V, 3, p ; k), \quad \text { for all } r \in(0,4 / 3] \text { and } p \in(0,1] .
$$

The smallest number of potential players when ex-post over-dissipation is possible is 4 :

$$
R T^{*}(5 / 4, V, 4,2 / 3 ; 4) \approx 1.049 V>V \text {. }
$$

Figures $4 \mathrm{a}$ and $4 \mathrm{~b}$ illustrate ex-post under-dissipation in the case of $n=3$ and ex-post over-

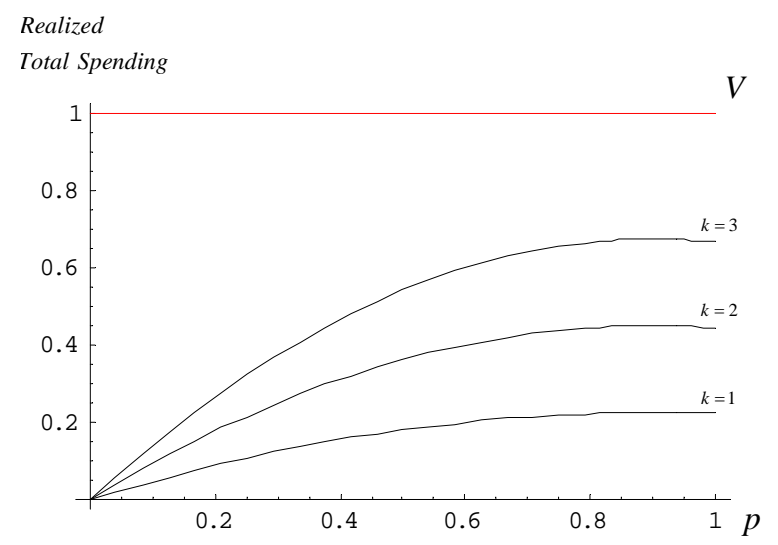

Figure 4: (a) realized total spending when $n=3$

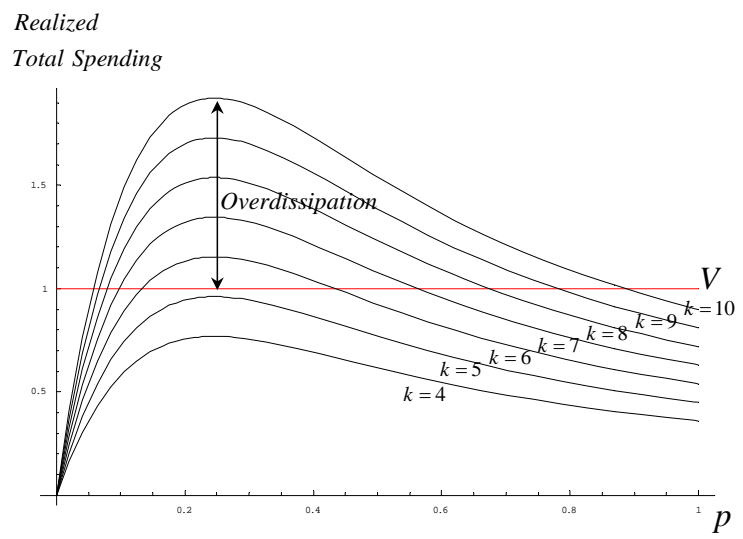

(b) realized total spending when $n=10$

dissipation in the case of $n=10$. We can see (Figures $4 \mathrm{~b}$ ) that ex-post over-dissipation can occur if $k \geq 6$. Moreover, the range of $p$ where ex-post over-dissipation can occur becomes wider as $k$ increases.

It is important to note that ex-post over-dissipation never takes place in the certain world because the actual number of players is the same as the expected number of players in this case. Therefore, some uncertainty must be present in order to have ex-post over-dissipation. This intuition is similar to the idea behind Baye, et al. $(1994,1999)$ who show that ex-post over-dissipation can take place as a particular realization of a mixed-strategy equilibrium. We summarize this subsection with the following theorem. 
Theorem 7. Ex-post over-dissipation takes place if and only if condition (15) holds. Moreover, if $n=2$ or 3 , ex-post over-dissipation never occurs; for any $n \geq 4$ there exist $p \in(0,1)$ and $r \in\left(0, \frac{n+1}{n}\right]$ such that ex-post over-dissipation is possible.

\subsection{The same expected number of players}

In this subsection, we fix the expected number of players, $\bar{n}=n p$, and compare total equilibrium spending across the total-equilibrium-spending curves in Figure 3. In particular, we are interested in comparing total equilibrium spending when $p=1$ and $n=\bar{n}, T^{*}(r, V, \bar{n}, 1)$,

with $0<p=\frac{\bar{n}}{n}<1$ and $n>\bar{n}, T^{*}\left(r, V, n, \frac{\bar{n}}{n}\right)$. Note that the expected number of players is the same in both cases.

From equations (6) and (13), we obtain

$$
T^{*}(r, V, \bar{n}, 1)=r V \frac{(\bar{n}-1)}{\bar{n}}
$$

and

$$
T^{*}\left(r, V, n, \frac{\bar{n}}{n}\right)=r V \cdot\left(\sum_{i=1}^{n} C_{i}^{n}\left(\frac{\bar{n}}{n}\right)^{i}\left(1-\frac{\bar{n}}{n}\right)^{n-i}\left(\frac{i-1}{i}\right)\right) .
$$

The following theorem presents the main result of this subsection that total equilibrium spending is strictly lower in any uncertain case in comparison with the certain case.

Theorem 8. Suppose that $\bar{n} \geq 2$. Then

$$
T^{*}(r, V, \bar{n}, 1)>T^{*}\left(r, V, n, \frac{\bar{n}}{n}\right),
$$

for any $n>\bar{n}$.

Theorem 8 shows that uncertainty about the actual number of players induces lower exante under-dissipation. This result is consistent with Myerson and Wärneryd's (2006) finding for another distribution of potential players. They consider a contest with infinitely many potential players where the number of players is a random variable with expectation $\mu>1$ and show that total equilibrium spending is strictly lower in such a contest than in a contest where the number of players is known with certainty to be $\mu$.

\section{Comparing Revealing and Concealing Policies}

In this section, we assume that a contest designer knows privately the realized number of players as it is in Levin and Ozdenoren (2004). Then, the risk-neutral contest designer has two different policies to consider: revealing his private information to the players or concealing 
it. Which policy is better for the contest designer who wants to maximize the expected total spending (which is equivalent to maximizing the expected individual spending in this case)? To answer this question, we consider the following two different scenarios. First, we assume that the contest designer can commit to always reveal or conceal the actual number of players (before he observes the actual number of players). We demonstrates that ex-ante the contest designer and the players are indifferent between whether the information about the actual number of participants in the contest is revealed or not. Second, we consider a strategic designer who can choose to either reveal or conceal the actual number of players after he obtains the information. We show that even if the contest designer can strategically decide whether to reveal the actual number of participating players or not, the actual number of participants is always revealed.

\section{$5.1 \quad$ Pre-commitment}

Suppose that the contest designer can commit himself to always either reveal or conceal his private information. Then, we have the following game:

1. The contest designer commits to reveal (subgame R) or conceal (subgame $\mathrm{C}$ ) his private information before the contest begins.

2. Nature chooses the number of active players.

3. The designer's commitment is implemented.

4. Active players choose their individual spending.

5. The winner of the game is determined by the contest success function (1).

Note that subgame $\mathrm{C}$ has already been considered in Section 2. In subgame $\mathrm{R}$, active players face Tullock's contest. The following result shows that ex-ante the contest designer and the players obtain the same expected payoffs in subgames $\mathrm{C}$ and $\mathrm{R}$.

Theorem 9. Ex-ante, both the contest designer and the players are indifferent between the revealing and the concealing policies.

This result follows from the expected utility theorem and the fact that individual equilibrium spending is a weighted average of individual equilibrium spending in case of $1,2,3, \ldots, n$ actual players, where the corresponding weights represent the binomial density assigned to the number of actual players.

\subsection{Strategic Designer}

What if the contest designer can strategically decide whether to reveal the actual number of participating players or not? To investigate this question, we consider the following game: 
1. Nature chooses the number of active players.

2. After observing the number of actual players, the contest designer decides either to reveal $(\mathrm{R}, \mathrm{i})$ or conceal $(\mathrm{C})$ it.

3. Active players choose their individual spending.

4. The winner of the game is determined by the contest success function (1).

Note that the contest designer's action at stage 2 depends on the realized actual number of players. Each player observes either $\mathrm{C}$ (subgame $\mathrm{C}$ ) or $(\mathrm{R}, \mathrm{i})($ subgame $(\mathrm{R}, \mathrm{i}))$ and makes her spending decision. Note that in the subgame $(\mathrm{R}, \mathrm{i})$ (the contest designer reveals that the actual number of players in the contest is $i$ ), Tullock's contest takes place and each active player spends

$$
\widehat{X}^{*}(R, i)=x(r, V, i)=\frac{i-1}{i^{2}} r V .
$$

In subgame $\mathrm{C}$, each player updates her belief about the actual number of players according to Bayes' rule. Denote by $f(j+1, p)$ the player's posterior belief that there are exactly $j$ opponents (or $j+1$ players including herself) in the contest. The analysis in Section 2 shows how to construct a unique symmetric equilibrium. Then, individual equilibrium spending is the weighted average:

$$
\widehat{X}^{*}(C)=\sum_{i=1}^{n} f(i ; p) x(r, V, i) .
$$

Let us look at the contest designer's decision problem. Given $\widehat{X}^{*}(R ; i)$ and $\widehat{X}^{*}(C)$, the contest designer reveals $i$ if

$$
\widehat{X}^{*}(R ; i)>\widehat{X}^{*}(C)
$$

From (9) and (17), the amount of individual spending as a function of the total number of players in the contest (the subgame $(\mathrm{R}, \mathrm{i})$ ) can be strictly ranked as follows:

$$
\widehat{X}^{*}(R, 2)>\widehat{X}^{*}(R, 3)>\ldots>\widehat{X}^{*}(R, n-1)>\widehat{X}^{*}(R, n)>\widehat{X}^{*}(R, 1)=0 .
$$

Therefore, $i=2$ is revealed in the equilibrium because $\widehat{X}^{*}(R ; 2)$ (the highest individual spending) is always greater than $\widehat{X}^{*}(C)$, which is a convex combination of some $\widehat{X}^{*}(R ; i)$ for $i \neq 2$. Since $i=2$ is revealed in the equilibrium (the designer chooses subgame $(\mathrm{R}, 2)$ ), there is zero probability to have two actual players in the contest in subgame $\mathrm{C}$, or $f(2 ; p)=0$. Therefore, $i=3$ should also be revealed in the equilibrium because $\widehat{X}^{*}(R ; 3)$ is now greater than $\widehat{X}^{*}(C)$, which is a convex combination of some $\widehat{X}^{*}(R ; i)$ for $i \neq 2,3$. Since $i=3$ is revealed in the equilibrium (the designer chooses the subgame $(\mathrm{R}, 3)$ ), there is zero probability of having three actual players in the contest in subgame $\mathrm{C}$, or $f(3 ; p)=0$. After applying the same logic to $i=4,5, \ldots . n$ recursively, we obtain the following result. 
Lemma 1. There is no symmetric perfect Bayesian equilibrium in which the contest designer conceals the number of active players $i>1$.

Lemma 1 demonstrates that in any symmetric perfect Bayesian equilibrium, either (a) the actual number of players is always revealed, or (b) only $i=1$ is concealed. It is important to note that concealing $i=1$ means that $f(1 ; p)=1$ and $f(i ; p)=0$ for all $i \neq 1$. Hence,

Theorem 10. In any symmetric perfect Bayesian equilibrium, full revelation takes place for any $p \in(0,1)$ and $n \geq 2$.

The last result is surprising because it demonstrates that the strategic designer cannot take advantage of his private information about the actual number of players.

\section{Conclusion}

In this paper, we consider Tullock's $n$-player contest where each player has an independent probability of participating. We find a unique symmetric equilibrium and analyze its properties. The most important observations are:

1. Even though individual equilibrium spending is not monotonic in the potential number of players and the participation probability, total equilibrium spending is monotonic in all parameters.

2. Ex-post over-dissipation is a natural feature of the unique symmetric pure-strategy equilibrium.

3. If the contest designer can privately learn the actual number of the contest participants, he will always reveal his private information.

There are several natural extensions of our paper. First, it would be interesting to test our theory in the experimental lab. In particular, our predictions about single-peak and singlecrossing properties of individual equilibrium spending and monotonicity of total equilibrium spending should be checked.

Finally, the actual number of players in our contest follows Binomial distribution, $B(n, p)$. This assumption captures the main source of the population uncertainty that results from the uncertainty each individual faces. Moreover, it is a well-known result that the binomial distribution converges towards the Poisson distribution with parameter $\lambda=n p$ as $n$ goes to infinity while the product $n p$ remains fixed. ${ }^{10}$ In this sense, we analyze a finite version of the "Poisson contest."

\footnotetext{
${ }^{10}$ See, for example, Feller (1968).
} 


\section{A Appendix: Proofs}

Proof of Theorem 1. In order to verify that (5) is indeed an equilibrium, we have to check the second order condition and confirm that individual spending (5) leads to a non-negative expected payoff for each player.

In the symmetric equilibrium, player $i$ 's expected payoff is

$$
V \cdot\left(\sum_{i=0}^{n-1} C_{i}^{n-1} p^{i}(1-p)^{n-i-1} \frac{(1-r) i+1}{(i+1)^{2}}\right) \geq 0 .
$$

It means that each player prefers to spend (5) instead of nothing, 0, given that each other player also spends (5). Condition (21) is equivalent to

$$
0<r \leq \frac{\sum_{i=0}^{n-1} C_{i}^{n-1} p^{i}(1-p)^{n-i-1} \frac{1}{(i+1)}}{\sum_{i=0}^{n-1} C_{i}^{n-1} p^{i}(1-p)^{n-i-1} \frac{i}{(i+1)^{2}}}
$$

The second order condition, from equation (3), is

$V \cdot\left(\sum_{\mathbf{M} \in \mathcal{P}_{i}} p^{|\mathbf{M}|}(1-p)^{\left|\mathbf{N}_{i} \backslash \mathbf{M}\right|} \frac{r \sum_{j \in \mathbf{M}} X_{j}^{r}}{\left(X_{i}^{r}+\sum_{j \in \mathbf{M}} X_{j}^{r}\right)^{3}}\left((r-1) X_{i}^{r-2}\left(X_{i}^{r}+\sum_{j \in \mathbf{M}} X_{j}^{r}\right)-2 r X_{i}^{2 r-2}\right)\right) \leq 0$.

In the symmetric equilibrium, this condition becomes

$$
0<r \leq \frac{\sum_{i=0}^{n-1} C_{i}^{n-1} p^{i}(1-p)^{n-i-1} \frac{i}{(i+1)^{2}}}{\sum_{i=0}^{n-1} C_{i}^{n-1} p^{i}(1-p)^{n-i-1} \frac{i(i-1)}{(i+1)^{3}}}
$$

Note that if $0<r \leq \frac{n+1}{n}$, then inequalities (22) and (23) always hold.

Proof of Theorem 2. Fix $n \geq 3$. Taking a derivative in (5) with respect to $p$ yields

$$
\begin{aligned}
\frac{1}{r V} \cdot \frac{\partial X^{*}(r, V, n, p)}{\partial p} & =\frac{(n-1)(1-p)^{n-2}}{4}+\sum_{i=1}^{n-2} \frac{(n-1) !}{i !(n-i-2) !} p^{i}(1-p)^{n-i-2}\left(\frac{i+1}{(i+2)^{2}}-\frac{i}{(i+1)^{2}}\right) \\
& =\frac{1}{r V} \cdot \frac{\partial X^{*}(r, V, n, p)}{\partial p}=(n-1)(1-p)^{n-2}\left(\frac{1}{4}-G(p, n)\right),
\end{aligned}
$$

where

$$
G(p, n)=\sum_{i=1}^{n-2} C_{i}^{n-2}\left(\frac{p}{1-p}\right)^{i}\left(\frac{i^{2}+i-1}{(i+1)^{2}(i+2)^{2}}\right)
$$

Note that

$$
\frac{1}{r V} \cdot \frac{\partial X^{*}(r, V, n, 0)}{\partial p}=\frac{(n-1)}{4}>0
$$

and since $n \geq 3$

$$
\frac{1}{r V} \cdot \frac{\partial X^{*}(r, V, n, 1)}{\partial p}=\frac{-n^{2}+3 n-1}{n^{2}(n-1)}=\frac{-n(n-3)-1}{n^{2}(n-1)}<0 .
$$


Since $\frac{1}{r V} \cdot \frac{\partial X^{*}(r, V, n, p)}{\partial p}$ is a continuous function of $p$ on the interval $[0,1]$, there must exist an interior $p^{*}(n) \in(0,1)$ such that $\frac{1}{r V} \cdot \frac{\partial X^{*}\left(r, V, n, p^{*}(n)\right)}{\partial p}=0$. Now, we shall show that $p^{*}(n)$ is unique.

Note that $G(p, n)>0$ for all $p \in[0,1]$ and function $G(p, n)$ is strictly increasing in $p$ since

$$
\frac{\partial}{\partial p} G(p, n)=\sum_{i=1}^{n-2} C_{i}^{n-2}\left(\frac{p}{1-p}\right)^{i-1} \frac{i}{(1-p)^{2}}\left(\frac{i^{2}+i-1}{(i+1)^{2}(i+2)^{2}}\right)>0 .
$$

It means that $G(p, n)$ is equal to $1 / 4$ only at the unique point $p^{*}(n)$. Therefore, there exists a unique $p^{*}(n)$ such that $\frac{1}{r V} \cdot \frac{\partial X^{*}\left(r, V, n, p^{*}(n)\right)}{\partial p}=0$. Finally, from equations $(24)$ and (26) it is clear that if $p<p^{*}(n)\left(p>p^{*}(n)\right)$, then $\frac{\partial X^{*}(r, V, n, p)}{\partial p}>0(<0)$. This completes the proof.

Proof of Theorem 3. From equation (5), we have

$$
\begin{gathered}
\frac{X^{*}(r, V, n, p)-X^{*}(r, V, n-1, p)}{r V}= \\
=p^{n-1} \frac{(n-1)}{n^{2}}+\sum_{i=1}^{n-2} C_{i}^{n-1} p^{i}(1-p)^{n-i-1} \frac{i}{(i+1)^{2}}-\sum_{i=1}^{n-2} C_{i}^{n-2} p^{i}(1-p)^{n-i-2} \frac{i}{(i+1)^{2}} \\
=p^{n-1} \frac{(n-1)}{n^{2}}+\sum_{i=1}^{n-2}\left(C_{i}^{n-2}+C_{i-1}^{n-2}\right) p^{i}(1-p)^{n-i-1} \frac{i}{(i+1)^{2}}-\sum_{i=1}^{n-2} C_{i}^{n-2} p^{i}(1-p)^{n-i-2} \frac{i}{(i+1)^{2}} \\
=\frac{p}{(n-1)}\left(\sum_{i=1}^{n-1} C_{i}^{n-1} p^{i-1}(1-p)^{n-i-1} \frac{i^{2}}{(i+1)^{2}}-\sum_{i=1}^{n-2} C_{i}^{n-1} p^{i}(1-p)^{n-i-2} \frac{i(n-i-1)}{(i+1)^{2}}\right) \\
=\frac{p}{(n-1)} \cdot \frac{1}{r V} \frac{\partial X^{*}(p, n)}{\partial p} .
\end{gathered}
$$

Proof of Theorem 4. First, we shall show that $p^{*}(n)$ strictly decreases as $n$ increases. From equation $(24)$, the unique optimizer $p^{*}(n)$ must satisfy

$$
G\left(p^{*}(n), n\right)=\frac{1}{4}
$$

Since

$$
\begin{gathered}
G(p, n+1)-G(p, n)= \\
\left(\frac{p}{1-p}\right)^{n-1}\left(\frac{n^{2}-n-1}{n^{2}(n+1)^{2}}\right)+\sum_{i=1}^{n-2} \frac{i}{n-i-1} C_{i}^{n-2}\left(\frac{p}{1-p}\right)^{i}\left(\frac{i^{2}+i-1}{(i+1)^{2}(i+2)^{2}}\right)>0
\end{gathered}
$$

for any $p \in(0,1)$ and $\frac{\partial G\left(p^{*}, n\right)}{\partial p^{*}}>0$, we get

$$
p^{*}(n)>p^{*}(n+1), \quad \text { for } n \geq 2 \text {. }
$$


Second, we shall show that function $X^{*}\left(r, V, n, p^{*}(n)\right)$ strictly decreases as $n$ increases. From Theorem 3, we have

$$
X^{*}\left(r, V, n, p^{*}(n)\right)=X^{*}\left(r, V, n-1, p^{*}(n)\right) .
$$

From (29), it follows that $p^{*}(n)<p^{*}(n-1)$. By the definition of $p^{*}(n-1)$ and its uniqueness, we get

$$
X^{*}\left(r, V, n-1, p^{*}(n-1)\right)>X^{*}\left(r, V, n-1, p^{*}(n)\right) .
$$

Therefore, from (30) and (31)

$$
X^{*}\left(r, V, n-1, p^{*}(n-1)\right)>X^{*}\left(r, V, n, p^{*}(n)\right) .
$$

Proof of Theorem 5. First, we show that two curves $X^{*}(r, V, m, p)$ and $X^{*}(r, V, n, p)$ intersect. Note that $G(0, n)=G(0, m)=0$. Therefore, from (24), we get

$$
\frac{\partial X^{*}(r, V, n, 0)}{\partial p}>\frac{\partial X^{*}(r, V, m, 0)}{\partial p}>0
$$

From (5) and (6), we have

$$
X^{*}(r, V, n, 0)=0=X^{*}(r, V, m, 0)
$$

and

$$
X^{*}(r, V, n, 1)=\frac{(n-1)}{n^{2}} r V<\frac{(m-1)}{m^{2}} r V=X^{*}(r, V, m, 1) .
$$

Continuity of $X^{*}(r, V, n, p)$ in $p$ together with (32), (33), and (34) provide the existence of an interior solution of equation (11). Now, we demonstrate that this interior solution is unique.

From Theorem 3, if $m=n-1$, then there exists a unique interior solution of equation (11), $p(n-1, n)=p^{*}(n)$. It means that for all $n>m \geq 2$ the following equations have a unique interior solution

$$
X^{*}\left(r, V, n, p^{*}(n)\right)=X^{*}\left(r, V, n-1, p^{*}(n)\right)
$$

and

$$
X^{*}\left(r, V, m, p^{*}(m+1)\right)=X^{*}\left(r, V, m+1, p^{*}(m+1)\right) .
$$

Moreover, from Theorem 4

$$
X^{*}(r, V, n, p)>X^{*}(r, V, n-1, p), \quad \text { if } \quad 0<p<p^{*}(n)
$$


and

$$
X^{*}(r, V, n, p)<X^{*}(r, V, n-1, p), \quad \text { if } \quad p>p^{*}(n) .
$$

Analogously,

$$
X^{*}(r, V, m, p)<X^{*}(r, V, m+1, p), \quad \text { if } \quad 0<p<p^{*}(m)
$$

and

$$
X^{*}(r, V, m, p)>X^{*}(r, V, m+1, p), \quad \text { if } \quad p>p^{*}(m) .
$$

Since $n>m$, Theorem 4 gives $p^{*}(n)<p^{*}(m)$. Therefore,

$$
X^{*}(r, V, n, p)>X^{*}(r, V, m, p), \quad \text { if } \quad 0<p<p^{*}(n)
$$

and

$$
X^{*}(r, V, n, p)<X^{*}(r, V, m, p), \quad \text { if } \quad p>p^{*}(m) .
$$

Hence, two curves $X^{*}(r, V, m, p)$ and $X^{*}(r, V, n, p)$ can cross only on the interval $p \in\left[p^{*}(n), p^{*}(m)\right]$. Note that function $X^{*}(r, V, m, p)$ is strictly increasing and function $X^{*}(r, V, n, p)$ is strictly decreasing on the interval $p \in\left[p^{*}(n), p^{*}(m)\right]$. Therefore, if there exists a solution of equation (11), it must be unique.

Proof of Theorem 6. First, we prove part i). Equation (13) yields

$$
\begin{gathered}
\frac{1}{r V n} \cdot \frac{\partial T^{*}(r, V, n, p)}{\partial p}=\sum_{i=1}^{n-1} C_{i}^{n-1} p^{i}(1-p)^{n-i-1} \frac{i}{(i+1)}-\sum_{i=1}^{n-2} C_{i}^{n-1} p^{i+1}(1-p)^{n-i-2} \frac{(n-i-1) i}{(i+1)^{2}} \\
=\frac{(n-1)}{2} p(1-p)^{n-2}+\sum_{i=1}^{n-2}\left(C_{i+1}^{n-1} p^{i+1}(1-p)^{n-i-2} \frac{(i+1)}{(i+2)}-C_{i}^{n-1} p^{i+1}(1-p)^{n-i-2} \frac{(n-i-1) i}{(i+1)^{2}}\right) \\
=\frac{(n-1)}{2} p(1-p)^{n-2}+\sum_{i=1}^{n-2} p^{i+1}(1-p)^{n-i-2} \frac{(n-1) !}{(i+1) !(n-i-2) !}\left(\frac{(i+1)}{(i+2)}-\frac{i}{(i+1)}\right) \\
=\left(\sum_{i=0}^{n-2} C_{i+1}^{n-1} p^{i+1}(1-p)^{n-i-2} \frac{1}{(i+1)(i+2)}\right)>0 .
\end{gathered}
$$

We prove part ii) now. From equation (13), we have

$$
\begin{gathered}
\frac{1}{r V}\left(T^{*}(r, V, k+1, p)-T^{*}(r, V, k, p)\right)= \\
p^{k+1} \frac{k}{(k+1)}+\sum_{i=1}^{k} C_{i}^{k+1} p^{i}(1-p)^{k-i+1} \frac{(i-1)}{i}-\sum_{i=1}^{k} C_{i}^{k} p^{i}(1-p)^{k-i} \frac{(i-1)}{i}=
\end{gathered}
$$




$$
\begin{gathered}
\sum_{i=1}^{k+1} C_{i-1}^{k} p^{i}(1-p)^{k-i+1} \frac{(i-1)}{i}-\sum_{i=1}^{k} C_{i}^{k} p^{i+1}(1-p)^{k-i} \frac{(i-1)}{i}= \\
\sum_{i=2}^{k+1} C_{i-1}^{k} p^{i}(1-p)^{k-i+1} \frac{1}{i(i-1)}>0 .
\end{gathered}
$$

Proof of Theorem 8. From equation (13), we get

$$
T^{*}(r, V, n, p)=r V \cdot\left(1-(1-p)^{n}-\sum_{i=1}^{n} C_{i}^{n} p^{i}(1-p)^{n-i} \frac{1}{i}\right) .
$$

Note that the function $\frac{1}{i}$ is strictly convex. By the Jensen's inequality, we have

$$
\begin{gathered}
\sum_{i=1}^{n} C_{i}^{n} p^{i}(1-p)^{n-i} \frac{1}{i}>\frac{\left(\sum_{i=1}^{n} C_{i}^{n} p^{i}(1-p)^{n-i}\right)^{2}}{\sum_{i=1}^{n} C_{i}^{n} p^{i}(1-p)^{n-i} i}= \\
=\frac{\left(\sum_{i=1}^{n} C_{i}^{n} p^{i}(1-p)^{n-i}\right)^{2}}{\bar{n}}=\frac{\left(1-(1-p)^{n}\right)^{2}}{\bar{n}} .
\end{gathered}
$$

Combining inequality (36) with equation (35), we obtain

$$
\begin{gathered}
T^{*}(r, V, n, p)<r V \cdot\left(1-(1-p)^{n}-\frac{\left(1-(1-p)^{n}\right)^{2}}{\bar{n}}\right)= \\
r V \frac{(\bar{n}-1)}{\bar{n}}+r V\left(\frac{2(1-p)^{n}}{\bar{n}}-(1-p)^{n}-\frac{(1-p)^{2 n}}{\bar{n}}\right)<T^{*}(r, V, \bar{n}, 1)-\frac{r V(1-p)^{2 n}}{\bar{n}} .
\end{gathered}
$$

The last inequality comes from the fact that $\bar{n} \geq 2$. This completes the proof.

\section{References}

[1] E. Baharad and S. Nitzan, Contest efforts in light of behavioral considerations, forthcoming in Economic Journal.

[2] M.R. Baye, D. Kovenock, and C.G. de Vries, The incidence of overdissipation in rentseeking contest, Public Choice 99 (1999) 439-454.

[3] M.R. Baye, D. Kovenock, and C.G. de Vries, The solution to the Tullock rent-seeking game when $R>2$ : Mixed-strategy equilibria and mean dissipation rates, Public Choice 81 (1994) 363-380.

[4] R. Congleton, A. Hillman and K. Konrad, Forty Years of Research on Rent Seeking: An overview, mimeo (2007). 
[5] W. Corcoran, Long-run equilibrium and total expenditures in rent-seeking. Public Choice 43 (1984) 89-94.

[6] W. Corcoran, and G. Karels, Rent-seeking behavior in the long-run. Public Choice 46 (1985) 227-247.

[7] W. Feller, An Introduction to Probability Theory and its Application, Vol. 1, third edn Wiley, New York (1968)

[8] L. Friedman, Game theory models of the allocation of advertising expenditures. Operations Research 6 (1958) 699-709.

[9] A. Hillman and D. Samet, Dissipation of contestable rents by small numbers of contenders. Public Choice 54 (1987) 63-82.

[10] R. Higgins, W. Shughart, and R. Tollison, Free entry and efficient rent seeking. Public Choice 46 (1985) 247-258.

[11] K. Konrad, Strategy in contests: An introduction, WZB Discussion Paper SP II 2007-01.

[12] A. Krueger, The political economy of the rent-seeking society, American Economic Review 64 (1974) 291-303.

[13] W. Leininger, and C. Yang, Dynamic rent-seeking games. Games and Economic Behavior 7 (1994) 406-427.

[14] D. Levin and E. Ozdenoren, Notes, Comments, and Letters to the Editor: Auction with Uncertain Numbers of Bidders, Journal of Economic Theory 118 (2004) 229-251.

[15] D. Levin and J. Smith, Equilibrium in Auctions with Entry, The American Economic Review 84 (1994) 585-599.

[16] B.G. Linster, A rent-seeking model of international competition and alliances. Defence Economics 4 (1993) 213-226.

[17] D. Malueg and A. Yates, Rent seeking with Private values, Public Choice 119 (2004) 161-178.

[18] R.P. McAfee and J. McMillan, Auctions with a stochastic number of bidders, Journal of Economic Theory 43 (1987) 1-19.

[19] R. Michaels, The design of rent-seeking competitions. Public Choice 56 (1988) 17-29. 
[20] J. Münster, Contests with an unknown number of contestants, Public Choice 129 (2006) 353-368.

[21] R. B. Myerson, Population uncertainty and Poisson games, International Journal of Game Theory 27 (1998) 375-392.

[22] R. B. Myerson and K. Wärneryd, Population uncertainty in contests, Economic Theory 27 (2006) 469-474.

[23] S. Nitzan, Modelling Rent-Seeking Contests, European Journal of Political Economy 10 (1994) 41-60.

[24] R. Posner, The social costs of monopoly and regulation, Journal of Political Economy 83 (1975) 807-827.

[25] S. Szymanski, The Economic Design of Sporting Contests, Journal of Economic Literature XLI (2003) 1137-1187.

[26] G. Tullock, Efficient rent-seeking. In J. M. Buchanan (Eds.), Toward a theory of the rent-seeking society, College Station, Texas, Texas A\&M University Press (1980) 99-112. 